\title{
A pulse skipping modulation with adaptive duty ratio in buck converter application
}

\author{
Dongjun Wang, Ping Luo ${ }^{\text {a)}}$, Qing Hua, \\ Shaowei Zhen, and Yajuan He \\ State Key Laboratory of Electronic Thin Films and Integrated Devices, \\ University of Electronic Science and Technology of China, Chengdu 610054, China \\ a)pingl@uestc.edu.cn
}

\begin{abstract}
A pulse skipping modulation (PSM) with adaptive duty ratio (APSM) in buck converter application is presented in this paper. The output voltage of converter is regulated by the APSM controller generating control pulse with multiple duty ratios. The duty ratio is approximately proportional to the square root of the voltage error between output voltage of converter and reference voltage. The experimental results and simulation are well consistent with theoretical analysis. The duty ratio can vary adaptively with the variance of voltage error and load. The APSM modulation technology can regulate output voltage slightly and improve ripple of output voltage especially in light load.
\end{abstract}

Keywords: PSM, buck converter, adaptive duty ratio, APSM

Classification: Integrated circuits

\section{References}

[1] A. Sinha and A. Chandrakasan: IEEE Des. Test Comput. 18 (2001) 62. DOI:10.1109/54.914626

[2] S. Kapat, S. Banerjee and A. Patra: IEEE Trans. Circuits Syst. I, Reg. Papers 57 (2010) 1793. DOI:10.1109/TCSI.2009.2034888

[3] S. Kapat, S. Banerjee and A. Patra: TENCON (2008) 1. DOI:10.1109/ TENCON.2008.4766640

[4] W. R. Liou and M. L. Kou: IEEE Trans. Power Electron. 23 (2008) 667. DOI:10.1109/TPEL.2007.915047

[5] P. Luo, L. Zhaoji and B. Zhang: ICCCAS 2 (2002) 1716. DOI:10.1109/ ICCCAS.2002.1179107

[6] P. Luo, L. Zhaoji and B. Zhang: ICCCAS 2 (2005) 1385. DOI:10.1109/ ICCCAS.2005.1495364

[7] S. Cliquennois, A. Donida, P. Malcovati, A. Baschirotto and A. Nagari: IEEE J. Solid-State Circuits 47 (2012) 1546. DOI:10.1109/JSSC.2012.2191214

[8] X. Gong, J. Jiang, X. Zhou and Z. Yuan: ASICON (2011) 838. DOI:10.1109/ ASICON.2011.6157335

[9] A. V. Peterche and S. R. Sanders: IEEE Trans. Power Electron. 21 (2006) 1588. DOI:10.1109/TPEL.2006.882968

[10] H. Li, B. Zhang and P. Luo: Analog Integr. Circ. Signal Process. 78 (2014) 361. DOI:10.1007/s10470-013-0231-7 


\section{Introduction}

Recently, alone with the rapid development of portable battery powered applications and emerging electronic devices like wearable devices and implantable medical electronic [1], the load of converter is becoming lighter and lighter, which results in output voltage and energy delivery of converter becoming finer and finer during each switching cycle $[2,3]$. Therefore, the large voltage ripple is actually becoming no longer acceptable from the perspective of application and efficiency $[4,5,6]$. To improve efficiency of converter in light load and sleep state, pulse skipping modulation (PSM) mode is adopted in converter [7, 8, 9]. For the PSM controlled buck converter, the output voltage $V_{\text {out }}$ of converter is regulated by a control pulse. If the $V_{\text {out }}$ is bigger than the reference voltage $V_{r e f}$ at the beginning of switching cycle, the power switch will be turned off by the control pulse with zero duty ratios and pulse skipping will occur; On the contrary, if $V_{\text {out }}<V_{\text {ref }}$, the power switch will be turned on by the control pulse with constant frequency and duty ratio $D_{\max }=30 \%$. From the perspective of energy, when converter works in discontinuous conduction mode (DCM), ignoring the power loss of power MOS, variation of energy in filtering inductor and capacitor, the total input energy $\Delta E_{I N}$ of converter during one switching cycle, can be expressed as

$$
\Delta E_{I N}=\int_{0}^{D_{A} T} V_{\text {out }} \frac{V_{\text {IN }}-V_{\text {out }}}{L} t d t=\frac{V_{\text {out }}\left(V_{\text {IN }}-V_{\text {out }}\right)}{2 L} D_{A}{ }^{2} T^{2}
$$

where $T, D_{A}, L$ and $V_{I N}$ are the switching cycle, the duty ratio of control pulse, the filtering inductance and input voltage, respectively [10]. The $\Delta E_{I N}$ reaches the maximum or zero when the $D_{A}$ is equal to $D_{\max }$ or zero, respectively. Hence, the voltage ripple of PSM controlled buck converter is a little bit bigger. As shown in Eq. (1), the ripple of output voltage can be improved via using the control pulse with multiple smaller duty ratios to regulate $V_{\text {out }}$. Therefore, the PSM with adaptive duty ratio (APSM) technique is proposed in this paper. For the APSM technique, multiple smaller duty ratios are generated adaptively by a simple circuit according to the different voltage error between output voltage and reference voltage. The output voltage can be regulated slightly and voltage ripple is also improved.

\section{Structure and working principle of proposed APSM technique}

As shown in Fig. 1, the block diagram of APSM controlled buck converter is given, it mainly includes the whole loop of buck converter, load resistance $R$ and APSM controller consisted of NAND gate, latches and voltage comparator. The output voltage $V_{\text {out }}$ of converter is applied on the $R$ and voltage comparator, and compared with reference voltage $V_{\text {ref }}$ in comparator. If $V_{\text {out }}>V_{\text {ref }}$, the comparison result $V_{C}$ will be logic high; On the contrary, if $V_{\text {out }}<V_{\text {ref }}$, the $V_{C}$ will be logic low.

In Fig. 2, the operating principle of APSM controller is shown. During the high level of $C L K$ at a switching cycle, if $V_{C}=1$ at the beginning of switching cycle, the power switch will be turned off and the $V_{\text {out }}$ will decrease because the charge of capacitor is consumed by the $R$. Once $V_{\text {out }}<V_{\text {ref }}\left(V_{C}=0\right)$ during high level of the same switching cycle, the power switch will be turned on immediately and be kept on in remaining of high level of this switching cycle, and the $V_{\text {out }}$ will increase. During high level of $C L K$ at a switch cycle, if $V_{C}=0$ at the beginning, the power 


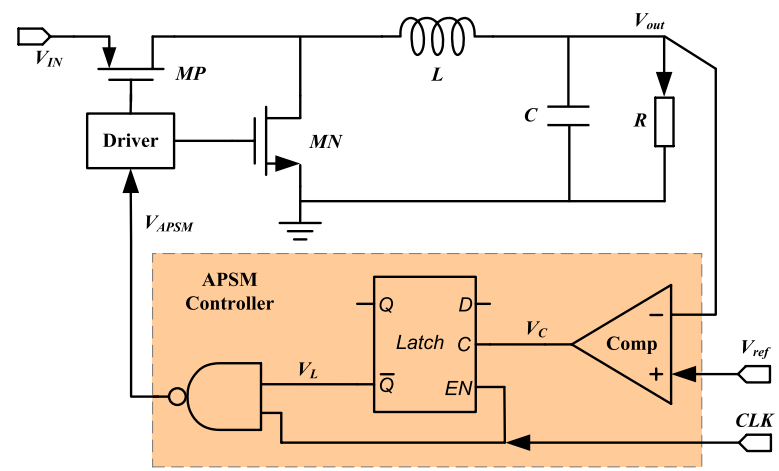

Fig. 1. The block diagram of APSM controlled buck converter

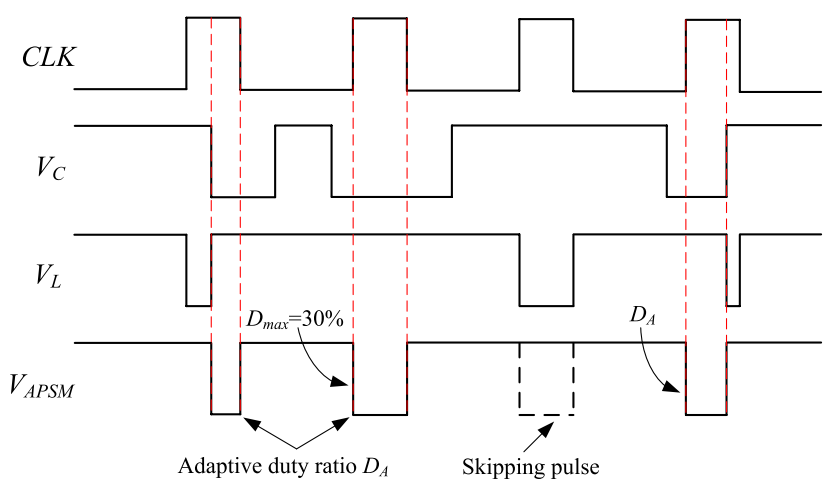

Fig. 2. The operating principle of APSM pulse generator

switch will be turned on and the $V_{\text {out }}$ will increase, once $V_{\text {out }}>V_{\text {ref }}\left(V_{C}=1\right)$, the power switch will be kept off in remaining of switching cycle. If $V_{C}=1$ during the high level of $C L K$, the pulse skipping will happen. So, the duty ratio of control pulse varies limberly with the variant output voltage $V_{\text {out }}$.

Now, the $D_{A}$ is defined as adaptive duty ratio (not zero or $D_{\max }$ ) in a switching cycle and voltage error $V_{e}=V_{\text {ref }}-V_{\text {out }}$. For the steady state of APSM controlled buck converter operating in DCM, the closely relationship between $D_{A}, R, \Delta V$ and $V_{e}$ in a switching cycle will be analyzed in detail. In Fig. 3, assuming output voltage $V_{o 1}$ is smaller than $V_{r e f}$ and inductor current $I_{L}$ is zero at the beginning of switching cycle. During time $n T$ to $\left(n+D_{A}\right) T$, the $I_{L}$ can be expressed as

$$
I_{L}=\left(V_{I N}-V_{\text {out }}\right)(t-n T) / L
$$

At time $t_{0}$, the $I_{L}$ equals to the load resistance current $I_{R}$, so

$$
I_{L}\left(t_{0}\right)=I_{R}=V_{\text {out }} / R
$$

The charge $Q_{1}$ flowing out from the filtering capacitor is

$$
Q_{1}=\left(t_{0}-n T\right) I_{R} / 2=C\left(V_{\text {ref }}-V_{o 1}\right)=V_{e} C
$$

At time $\left(n+D_{A}\right) T$, the peak current of inductor $I_{P}$ is

$$
I_{P}=\left(V_{I N}-V_{\text {out }}\right)\left(D_{A} T\right) / L
$$

During time $t_{0}$ to $\left(n+D_{A}\right) T$, the charge $Q_{2}$ flowing into the filtering capacitor is

$$
Q_{2}=\left[\left(n+D_{A}\right) T-t_{0}\right]\left(I_{P}-I_{R}\right) / 2=C\left(V_{\text {ref }}-V_{o 2}\right)=\Delta V_{2} C
$$

The voltage error $V_{e}$ can be expressed as 


$$
V_{e}=V_{r e f}-V_{o 1}=\Delta V_{2}-\Delta V_{1}=\left(Q_{2}-Q_{1}\right) / C
$$

Substituting Eqs. (2)-(6) into Eq. (7), the duty ratio $D_{A}$ can be derived as

$$
D_{A}=\frac{\sqrt{2 C L\left(V_{\text {IN }}-V_{\text {out }}\right) V_{e}+\left(\frac{L V_{\text {out }}}{R}\right)^{2}}+\frac{L V_{\text {out }}}{R}}{T\left(V_{\text {IN }}-V_{\text {out }}\right)}
$$

As shown in Eq. (8), when the $R$ keeps constant, the $D_{A}$ will be larger if the $V_{e}$ becomes larger, but the maximum of $D_{A}$ is restricted by the maximum duty ratio $D_{\max }$ and equals to $D_{\max }$. As the $V_{e}$ keeps fixed, the $D_{A}$ will be smaller with the $R$ becoming larger and the minimum of which is $D_{A \min }=2 L V_{\text {out }} /\left[R T *\left(V_{I N}-V_{\text {out }}\right)\right]$.

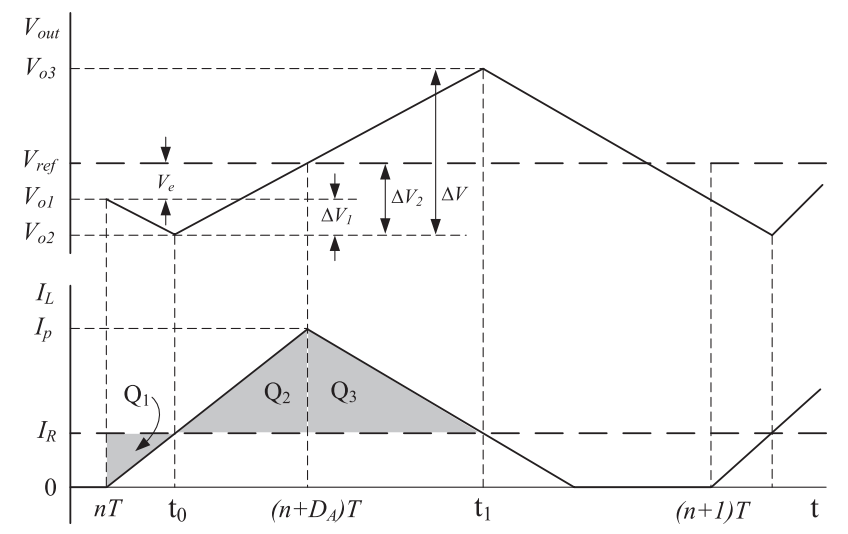

Fig. 3. The relationship between adaptively duty ratio $D_{A}$ and voltage error $V_{e}$

During time $\left(n+D_{A}\right) T$ to $t_{1}$, the inductor current $I_{L}$ can be written as

$$
I_{L}=I_{P}-\left[t-\left(n+D_{A}\right) T\right] V_{\text {out }} / L
$$

At time $t_{1}$, the $I_{L}$ equals to the load resistance current $I_{R}$, so

$$
I_{L}\left(t_{1}\right)=I_{R}=V_{\text {out }} / R
$$

The charge $Q_{3}$ flowing into the filtering capacitor during time $\left(n+D_{A}\right) T$ to $t_{1}$ is

$$
Q_{3}=\left[t_{1}-\left(n+D_{A}\right) T\right]\left(I_{P}-I_{R}\right) / 2
$$

The ripple of output voltage $\Delta V$ can be expressed as

$$
\Delta V=V_{o 3}-V_{o 2}=\left(Q_{3}+Q_{2}\right) / C
$$

Substituting Eqs. (2)-(6) and Eqs. (9)-(11) into Eq. (12), the $\Delta V$ can be derived as

$$
\Delta V=\frac{1}{2 L C} \frac{V_{I N}}{V_{\text {out }}}\left(D_{A} T\right)^{2}\left(V_{\text {IN }}-V_{\text {out }}\right)\left(1-\frac{L V_{\text {out }}}{D_{A} T R\left(V_{I N}-V_{\text {out }}\right)}\right)^{2}
$$

In Eq. (13), when the $R$ keeps fixed, the larger $D_{A}$ is, the larger $\Delta V$ is. For the lighter load, the $\Delta V$ will be larger if the $D_{A}$ keeps constant. According to Eq. (8) and Eq. (13), the $D_{A}$ is approximately proportional to the square root of the $V_{e}$, and varies adaptively with the variance of $V_{e}$. If $V_{\text {out }}$ is dropped largely in a flash, namely the $V_{e}$ is larger, the $D_{A}$ will be larger immediately, which can speed up the transient response of converter and result in larger voltage ripple. The lighter load is, the smaller $D_{A}$ is, and the $\Delta V$ is also smaller. So, if $V_{\text {out }}>V_{\text {ref }}$, the duty ratio $D_{A}$ 
of control pulse is zero. Otherwise the duty ratio $D_{A}$ is adaptively with the voltage error $V_{e}$ variance. Compared to fixed duty ratio of traditional PSM mode, the APSM technique can improve voltage ripple and the transient response speed by theoretical analysis.

\section{Simulation and experimental results}

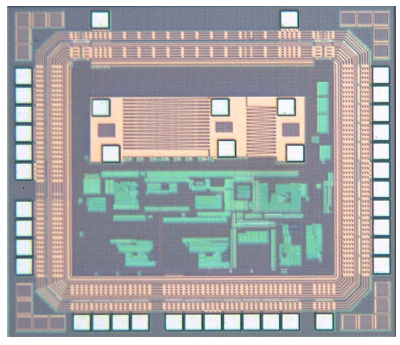

Fig. 4. Die micrograph

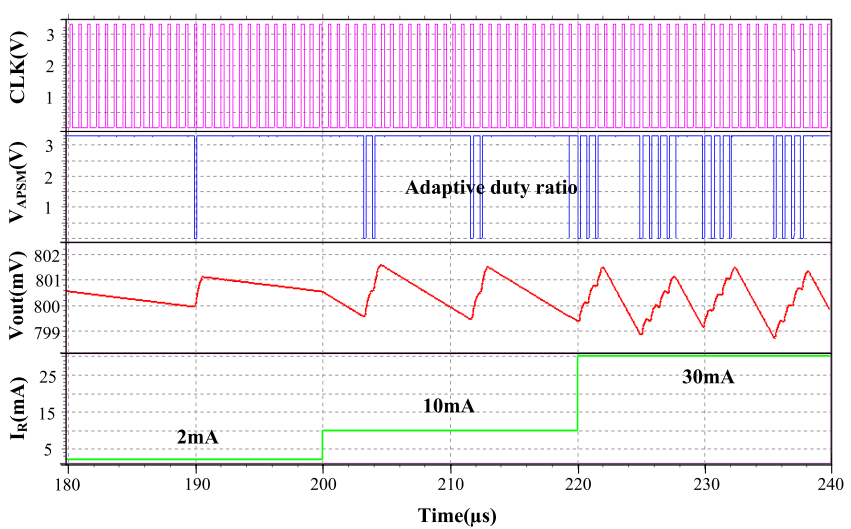

Fig. 5. Simulation of APSM controlled buck converter operating in different load

The proposed APSM technique in buck converter application in this paper is fabricated in a standard $0.13 \mu \mathrm{m}$ CMOS process. The die micrograph is shown in Fig. 4. The main modules shown in Fig. 1 are integrated on the chip, including the power MOS and the driver, and the frequency and duty ratio of clock $C L K$ generated on the chip are $1.5 \mathrm{MHz}$ and $30 \%$, respectively. The external devices only include filtering inductor $L$ and filtering capacitor $C$, which are designed as $3.3 \mu \mathrm{H}$ and $22 \mu \mathrm{F}$, respectively. The input voltage $V_{I N}$ is $3.3 \mathrm{~V}$ during simulation and measurement. The simulation result of APSM controlled buck converter operating in different loads is shown in Fig. 5. The simulation results shows voltage ripple decreases with load becoming lighter, the adaptive duty ratio and pulse skipping are observed obviously. In Fig. 6, the measured waveform of APSM controlled buck converter in steady state is shown. The output voltage $V_{\text {out }}$ is regulated by the control pulse with adaptive duty ratio. The $V_{\text {out }}$ is $0.8 \mathrm{~V}$ and the voltage ripple is about $50 \mathrm{mV}$, and the adaptive duty ratio is also observed obviously. Therefore, the simulation and experimental results correspond well to the theoretical analysis. 


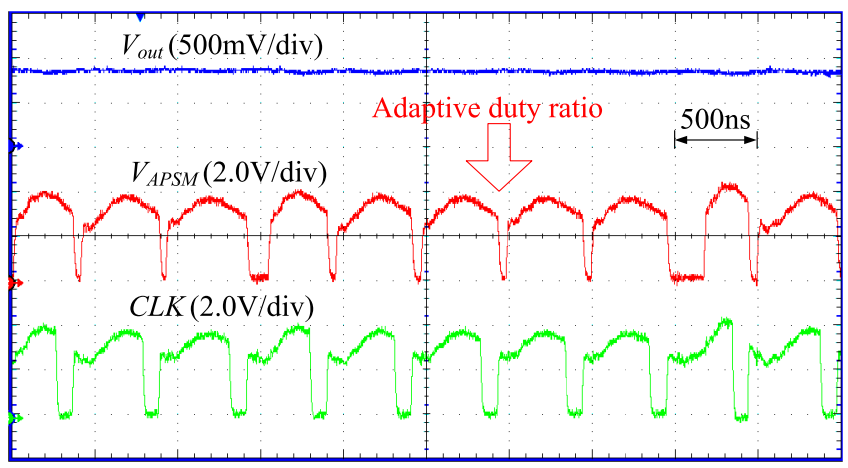

Fig. 6. Measured waveform of APSM controlled buck converter in steady state

\section{Conclusion}

The APSM technique in buck converter application is presented in this paper. By the theoretical analysis, the relationship between duty ratio, voltage error, load and output voltage ripple are expressed. The duty ratio is approximately proportional to the square root of the voltage error, and duty ratio varies adaptively with the variant voltage error and load. The output voltage is scaled by the control pulse with adaptive duty ratios, and voltage ripple is improved.

\section{Acknowledgments}

This work was supported in part by the National Natural Science Foundation of China under Grant 61274027, the National Natural Science Foundation of China under Grant 61404025 and the Fundamental Research Funds for the Central Universities under Grant ZYGX2014J024. 